\title{
Geotail observations of FTE velocities
}

\author{
G. I. Korotova ${ }^{1,2}$, D. G. Sibeck ${ }^{3}$, and T. Rosenberg ${ }^{2}$ \\ ${ }^{1}$ IZMIRAN, Troitsk, Moscow Region 142190, Russia \\ ${ }^{2}$ IPST, UMD, College Park, MD 20742, USA \\ ${ }^{3}$ Code 674, NASA/GSFC, Greenbelt, MD 20771, USA
}

Received: 29 December 2007 - Revised: 7 October 2008 - Accepted: 5 November 2008 - Published: 6 January 2009

\begin{abstract}
We discuss the plasma velocity signatures expected in association with flux transfer events (FTEs). Events moving faster than or opposite the ambient media should generate bipolar inward/outward (outward/inward) flow perturbations normal to the nominal magnetopause in the magnetosphere (magnetosheath). Flow perturbations directly upstream and downstream from the events should be in the direction of event motion. Flows on the flanks should be in the direction opposite the motion of events moving at subsonic and subAlfvénic speeds relative to the ambient plasma. Events moving with the ambient flow should generate no flow perturbations in the ambient plasma. Alfvén waves propagating parallel (antiparallel) to the axial magnetic field of FTEs may generate anticorrelated (correlated) magnetic field and flow perturbations within the core region of FTEs. We present case studies illustrating many of these signatures. In the examples considered, Alfvén waves propagate along event axes away from the inferred reconnection site. A statistical study of FTEs observed by Geotail over a 3.5-year period reveals that FTEs within the magnetosphere invariably move faster than the ambient flow, while those in the magnetosheath move both faster and slower than the ambient flow.
\end{abstract}

Keywords. Interplanetary physics (Solar wind plasma) Magnetospheric physics (Solar wind-magnetosphere interactions) - Space plasma physics (Magnetic reconnection)

\section{Introduction}

Magnetic reconnection dominates the solar windmagnetosphere interaction. During periods of southward interplanetary magnetic field (IMF) orientation, reconnection removes magnetic flux from the dayside magnetosphere, advects it across the polar cap, and deposits it in the Earth's

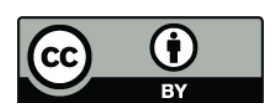

Correspondence to: G. I. Korotova (korotova@excite.com) magnetotail (Dungey, 1961). Reconnection on the dayside magnetopause may be steady or bursty, localized or widespread (Cowley, 1982). Bursty and patchy reconnection generates flux tubes of interconnected magnetosheath and magnetospheric magnetic field lines (Russell and Elphic, 1978). Bursty reconnection along extended single $x$-lines generates bubbles of interconnected magnetosheath and magnetospheric magnetic field lines (Southwood et al., 1988; Scholer, 1988a), whereas bursty reconnection along extended parallel $\mathrm{x}$-lines generates flux ropes with spiraling magnetic fields (Lee and $\mathrm{Fu}, 1985$ ). In component reconnection models (e.g. Sonnerup, 1974), the tilt of the reconnection line(s) depends upon the IMF orientation, so that the axes of the bubbles or flux ropes should (at least initially) run from northern dawn to southern dusk during periods of dawnward IMF orientation, but from southern dawn to northern dusk during periods of duskward IMF orientation.

Reconnection converts energy stored within the magnetic field into plasma jets whose subsequent thermalization causes the bubbles/flux ropes to swell outward. As the events expand, they compress and distort magnetic field lines in the neighboring magnetosheath and magnetosphere. When magnetosheath and magnetospheric magnetic fields lie nearly antiparallel, theory and simulations predict magnetic field strengths within the core regions of the bubbles/flux ropes far less than those in the nearby magnetosphere and magnetosheath (Ding et al., 1991). When the shear angle between magnetosheath and magnetospheric magnetic fields is finite, the newly reconnected magnetic field lines sweep up field lines in their path, resulting in core regions with larger magnetic field strengths (Scholer, 1988b).

The newly reconnected magnetic field lines within bubbles and flux ropes move under the influence of pressure gradient and magnetic curvature forces (e.g. Cooling et al., 2001). Farrugia et al. (1987) treated the case of incompressible single-adiabatic flow and magnetic field draping about rigid elongated cylindrical FTEs with arbitrary elliptical crosssections moving along the magnetopause boundary between

Published by Copernicus Publications on behalf of the European Geosciences Union. 
magnetosheath and magnetospheric plasmas, while Sonnerup et al. (1992) considered compressible single/doubleadiabatic flows and magnetic field draping about slender cylinders that do not protrude far from the magnetopause. Spacecraft that remain outside passing events observe transient enhancements in the magnetic field strength and bipolar magnetic field perturbations in the direction normal to the nominal magnetopause, with the sense of the bipolar perturbation depending upon the direction of event motion relative to the external magnetic field (Rijnbeek et al., 1982).

The draped magnetic field rotates towards the direction perpendicular to the event axis during the passage of events moving through incompressible plasmas and events moving slowly through compressible plasmas, but towards the axis for events moving through the compressible plasmas at Mach numbers satisfying $\mathrm{M}_{S}^{2}+\mathrm{M}_{A}^{2}>1$, where $\mathrm{M}_{S}$ and $\mathrm{M}_{A}$ are the sonic and Alfvénic Mach numbers. Densities and temperatures remain constant in incompressible plasmas. The situation in compressible single-adiabatic plasmas, is somewhat more complicated. When the component of the magnetic field perpendicular to the event axis is large, densities decrease for subsonic flows and increase for supersonic flows. When the component of the magnetic field perpendicular to the event axis is small, densities decrease for slowly-moving events, decrease for moderately superAlfvénic events, and increase for subAlfvénic events moving through the plasma at Mach numbers satisfying $\mathrm{M}_{S}^{2}+\mathrm{M}_{A}^{2}-$ $\mathrm{M}_{S}^{2} \mathrm{M}_{A}^{2} B_{\perp}^{2} / B^{2}>1$, where $B_{\perp}$ is the component of the magnetic field perpendicular to the event axis. In both the perpendicular and parallel cases, density variations are relatively small except in the immediate proximity of the transition zones. In double-adiabatic plasmas, densities and temperatures increase during the passage of events moving at both subsonic and supersonic speeds relative to the ambient flow, where the sound speed is based on the temperature parallel to the magnetic field.

Spacecraft entering the events may also observe bipolar magnetic field signatures normal to the nominal magnetopause as a result of the sheared magnetosheath and magnetospheric magnetic fields from which they are formed (e.g. Lee and $\mathrm{Fu}, 1985$ ) or due to Alfvén waves propagating along event axes (Saunders et al., 1984). Magnetic field strengths within the core region of FTEs generated by anti-parallel reconnection should be greatly depressed, while those within the core region of events generated by component reconnection should may be only slightly depressed or even enhanced. Spacecraft entering the core region must observe intermediate densities and temperatures as well as a mixture of magnetosheath and magnetospheric plasmas, since the events contain interconnected magnetic field lines. Since magnetosheath densities are greater and temperatures lower than those in the magnetosphere, spacecraft entering the core region of FTEs do not observe the correlated density and temperature variations predicted outside the events.
Observations in the vicinity of the dayside magnetopause during periods of southward IMF orientation indicate that events marked by bipolar magnetic field signatures normal to the nominal magnetopause and enhanced (Russell and Elphic, 1978) or crater-like (Rijnbeek et al., 1987) magnetic field strength variations are common. Features with these characteristics are called flux transfer events (FTEs). They are believed to have dimensions on the order of $\sim 1 R_{E}$ in the direction normal to the magnetopause and $\sim 2 R_{E}$ in the direction of motion along the magnetopause (Saunders et al., 1984). Their azimuthal extent along the magnetopause in the direction transverse to their motion cannot be determined from in situ observations by single or multiple closely-spaced spacecraft at the magnetopause, but estimates made on the basis of ground observations suggest an extent greater than $4 \mathrm{~h}$ in local time (Lockwood et al., 1990), corresponding to $\sim 10 R_{E}$ at the magnetopause.

FTEs also generate transient plasma flow signatures, whose characteristics depend upon event dimension, velocity, orientation, and internal structure. This paper begins by outlining the flow perturbations expected for FTEs. We then present case studies illustrating the range of signatures observed and a statistical survey describing the occurrence patterns of these signatures on the surface of the magnetopause.

\section{FTE flow signature predictions}

FTEs generate characteristic flow signatures as they move along the magnetopause. These flow signatures depend upon the velocities of the events relative to the surrounding media. Here we consider events moving at subsonic and subAlfvénic flow speeds relative to the ambient media. It is most convenient to consider the flow perturbations in the boundary normal coordinate system presented by Russell and Elphic (1978), where $\boldsymbol{n}$ points outward perpendicular to the model magnetopause, $\boldsymbol{l}$ points northward in the plane of the magnetopause, and $\boldsymbol{m}$ completes the triad by pointing dawnward in the plane of the magnetopause.

Consider first the flow perturbations produced by an event moving faster than the ambient flows in either adjacent region (Sibeck and Smith, 1992; Ku and Sibeck, 1998). Figure 1 shows an FTE moving dawnward (+m direction) along the subsolar magnetopause at a speed $+V_{m}$ (large red arrow). There is no background flow in either the magnetosheath or the magnetosphere. The event pushes the plasma in its path ahead, entrains it behind (large black arrows), and displaces it on the flanks. The sense of the resulting perturbations is away from the event on the leading edge, opposite event motion on the flanks, and toward the event on the trailing edge. Solid black curves illustrate streamlines in the rest frame of the ambient plasmas, while the black arrows indicate the sense of the flow perturbations in these neighboring regions.

Consequently, magnetosheath spacecraft in the path of the FTE (A and B) should observe outward/inward $(+,-)$ flows 


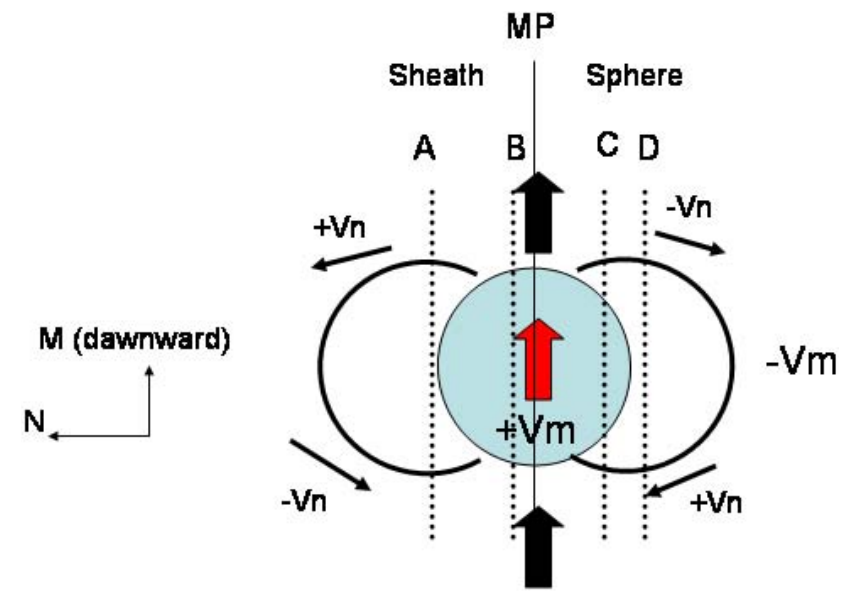

Fig. 1. An FTE moving dawnward on the magnetopause at a speed $+V_{m}$ (red arrow) that is greater than those of the ambient magnetosheath and magnetospheric plasma. Plasma in front of the event is pushed forward, while that behind is entrained (bold black arrows). The event pushes the surrounding plasma to the side, generating flows opposite its motion on its flanks (thin black arrows). Spacecraft that remain in the magnetosheath (A) observe outward/inward $(+,-)$ velocities normal to the nominal magnetopause $\left(V_{n}\right)$, while those that remain in the magnetosphere (D) observe inward/outward $(-,+)$ velocities. Spacecraft that make glancing encounters $(\mathrm{C})$ may observe tripolar flow signatures: $+V_{m}$ ahead of the event, $-V_{m}$ on the flanks of the event, and $+V_{m}$ on the trailing edge of the event. Spacecraft that make direct encounters with the event (B) observe flows in the $+V_{m}$ direction throughout the encounter. As there are no Alfvén waves within the FTE, there are no flows normal to the nominal magnetopause within the event.

in the direction normal to the nominal magnetopause $(n)$, while magnetospheric spacecraft in the path of the FTE (C and D) should observe inward/outward flows. Spacecraft that remain outside the event (A and $\mathrm{D}$ ) observe strong flows in the direction $(-m)$ opposite its motion. Spacecraft making glancing entries into the event $(\mathrm{C})$ may observe tripolar flow signatures in the direction along the magnetopause. Outside the leading edge of the event they observe flows opposite event motion $\left(-V_{m}\right)$, within the core they observe flows in the direction of event motion, and then outside the trailing edge of the event they again observe flows in the direction opposite event motion. Spacecraft passing directly through the core region of the event (B) observe flows in the direction of event motion at all times. All of these signatures can be seen in the MHD simulations of FTEs produced by the onset of reconnection along extended reconnection lines in the presence and absence of background magnetosheath flows presented by Ku and Sibeck $(1997,1998)$.

Now consider the signatures produced by an event moving with the magnetosheath flow. As illustrated in Fig. 2, in the absence of any motion relative to the background magnetosheath flow, spacecraft on the magnetosheath side (E) observe no flow signatures. However the event elicits bipo-

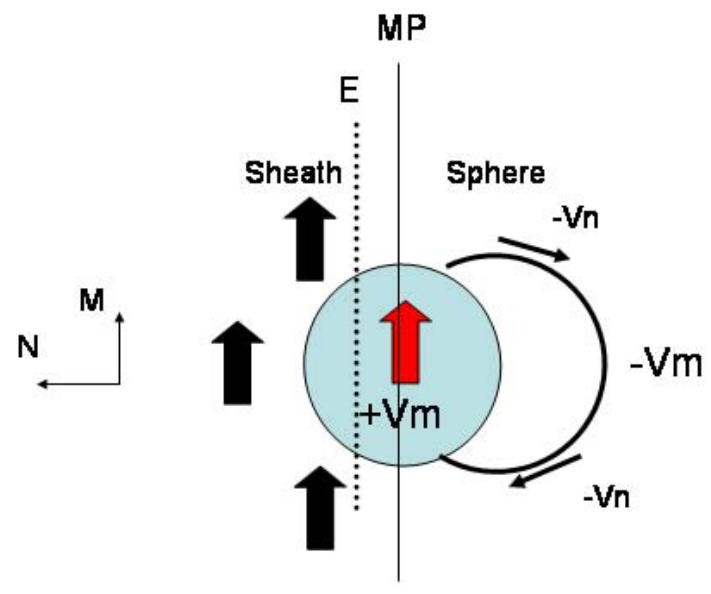

Fig. 2. An FTE moving dawnward on the magnetopause with the magnetosheath flow $\left(+V_{m}\right)$, which is greater than that in the magnetosphere. As the event and magnetosheath plasma move at the same speed, and the event is not rotating, spacecraft in the magnetosheath (E) observe no flow perturbations whatsoever. By contrast, spacecraft in the magnetosphere should observe signatures similar to those described in Fig. 1.

lar inward/outward flows and a flow opposite the direction of its motion in the nearby magnetosphere, just as in the case considered earlier.

Finally, consider an event moving in the direction of the magnetosheath flow, but at a speed slower than that of the ambient flow. As illustrated in Fig. 3, the event poses an obstacle to the flow, which moves over and around the event. A spacecraft (F) initially within the magnetosheath that remained outside the event during its passage would observe inward/outward $V_{n}$ flows centered on an accelerated flow in the direction of event motion $\left(+V_{m}\right)$. The pattern of flows within the magnetosphere, where there is no background flow, resembles that in the previous cases.

There is reason to believe that events revolve about their axes. Saunders et al. (1984) attributed flows normal to the nominal magnetopause within the core region of events to Alfvén waves propagating along their axes. If so, the observed velocities will be the sum of those associated with the translation and rotation. In the absence of rotation, no flows normal to the nominal magnetopause will be observed within the core region of the events. Figure 4 illustrates the case when an event moves northward ( $l$ direction) slowly (thin red arrow) while revolving rapidly about its axis (thick curved red arrows). Spacecraft in the magnetosheath that enter the event $(\mathrm{G})$ observe inward/outward $\left(-,+V_{n}\right)$ flows centered on a $+V_{l}$ flow within the core region of the event. Spacecraft in the magnetosphere that enter the event $(\mathrm{H})$ also observe inward/outward $\left(-,+V_{n}\right)$ flows, but centered on a $-V_{l}$ flow. The sense of the inward/outward flows, and the flows along the magnetopause, should reverse for an event rotating in the opposite sense, i.e. for an Alfvén wave 


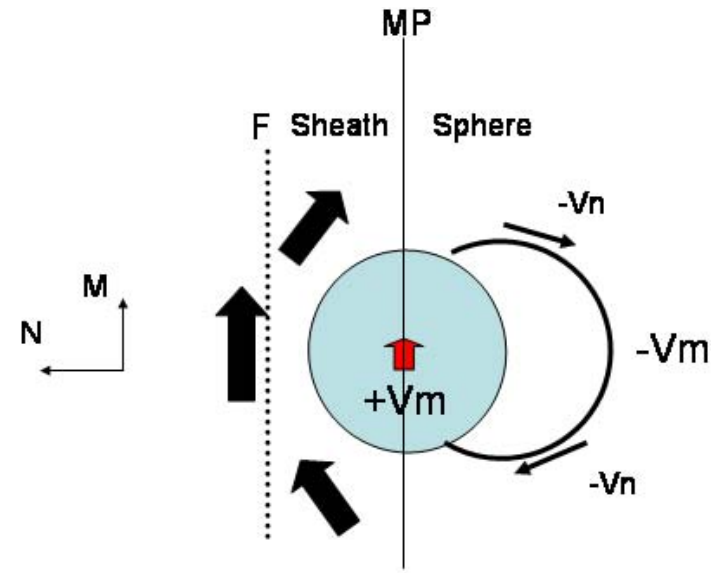

Fig. 3. An FTE moving dawnward on the magnetopause at a speed $\left(+V_{m}\right)$ slower than that of the magnetosheath, but faster than that of the ambient magnetospheric plasma. Because the magnetosheath plasma flows over the event, spacecraft that remain within the magnetosheath during its passage (F) observe inward/outward $(-,+) V_{n}$ signatures normal to the nominal magnetopause and enhanced flows in the direction of event motion. As the event is not rotating, spacecraft entering it from the magnetosheath side of the magnetopause should observe diminished flows in the direction of event motion. Spacecraft that remain within the magnetosphere observe flows similar to those described in Fig. 1.

propagating in the opposite direction along the event axis. The amplitudes of the signatures observed depend upon the impact parameter. The bipolar flows normal to the nominal magnetopause reach greatest amplitudes along paths that pass near the center of the event (I), whereas the flows along the magnetopause reach greatest amplitudes on paths that pass near the outermost edge of the event $(\mathrm{H})$. Note that the sense of the $V_{n}-B_{n}$ correlation indicates the direction of Alfvén wave propagation: negative indicates propagation along the direction of the core magnetic field, while positive indicates propagation opposite (Belcher and Davis, 1971).

Had the event shown in Fig. 4 been moving along the magnetopause in the $+l$-direction at a speed greater than that associated with the rotation, velocities throughout the core region of the event would have been in the $+l$ direction, but larger on the magnetosheath side (where the event and rotational velocities would have been in the same direction) than on the magnetospheric side (where the event and rotational velocities would have been opposed). Flows outside the events would have been like those shown in Fig. 1. Consequently, the sense (if not the magnitude) of the $V_{n}$ signatures would have remained constant across the event boundary. Had the event been rotating in the opposite direction, neither the sense nor the magnitude of the $V_{n}$ signatures would have remained constant across its boundaries.

Table 1 summarizes the taxonomy of FTE flow signatures possible for events moving at subsonic and subAlfvénic velocities relative to the ambient media.

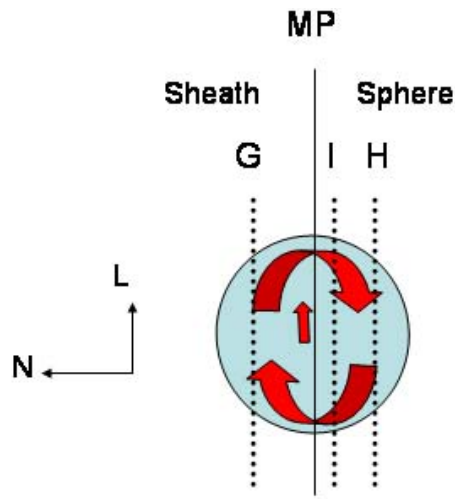

Fig. 4. An event rotating about its axis as it moves slowly northward $\left(+V_{l}\right)$ on the magnetopause. A spacecraft in the magnetosheath $(\mathrm{G})$ observes inward/outward $(-,+) V_{n}$ flows centered on a northward $\left(+V_{l}\right)$ flow as the event passes. While a spacecraft in the magnetosphere $(\mathrm{H})$ also observes inward/outward $V_{n}$ flows, they are centered on a southward $\left(-V_{l}\right)$ flow. A spacecraft in the magnetosphere that is closer to the magnetopause (I) observes stronger bipolar inward/outward flows, but weaker southward flows inside the event.

\section{Data set and orbit}

The Geotail spacecraft was launched on 24 July 1992. Since February 1995, the spacecraft has been in an equatorial orbit with a $\sim 10 R_{E}$ perigee and a $30 R_{E}$ apogee. This study employs $3 \mathrm{~s}$ time resolution magnetic field (Kokubun et al., 1994) and $12 \mathrm{~s}$ time resolution low-energy plasma (LEP) (Mukai et al., 1994) moment observations by Geotail from February 1994 to June 1997. Figure 5 presents the projection of the Geotail orbit during 1996 into the GSM X-Y plane to illustrate the range of locations where magnetopause crossings and FTEs might be observed.

FTEs are most readily identified on the basis of their bipolar magnetic signatures normal to the nominal magnetopause. We plotted the Geotail plasma and magnetometer observations in boundary normal coordinates using the Roelof and Sibeck (1993) model magnetopause for nominal solar wind conditions (solar wind dynamic pressure $=2 \mathrm{nPa}$, IMF $B_{z}=0$ ) to determine the normal to the nominal magnetopause. We identified FTEs on the basis of their magnetic field signatures alone, namely:

1. FTEs must exhibit clear symmetrical bipolar signatures in the magnetic field component $\left(B_{n}\right)$ normal to the nominal magnetopause with peak-to-peak amplitudes exceeding $4 \mathrm{nT}$.

2. FTEs must exhibit either a monopolar or a crater-like enhancement in the total magnetic field strength.

3. Events with bipolar signatures centered on magnetopause crossings and events in which the bipolar $\mathrm{B}_{n}$ variations do not lie centered on peaks (or craters) in the magnetic field strength are excluded from our study. 
Table 1. Flow signature taxonomy and interpretation.

\begin{tabular}{|c|c|c|c|}
\hline Region observed & $V_{n}$ signature & $\delta V_{l m}$ signature & Interpretation \\
\hline Magnetosheath &,+- & $\begin{array}{l}\text { Opposite direction of event motion } \\
\text { inferred from } B_{n} \text { signature }\end{array}$ & $V_{\text {event }}>V_{\text {sheath }}$ \\
\hline Magnetosheath & None & None & $V_{\text {event }}=V_{\text {sheath }}$ \\
\hline Magnetosheath &,-+ & $\begin{array}{l}\text { In direction of event motion inferred } \\
\text { from } B_{n} \text { signature }\end{array}$ & $V_{\text {event }}<V_{\text {sheath }}$ \\
\hline Core & $\begin{array}{l}+,- \\
-,+\end{array}$ & $\begin{array}{l}\text { Sum of rotation and } \\
\text { translation velocities }\end{array}$ & $\begin{array}{l}\text { Alfvén wave anti // } B \\
\text { Alfvén wave // } B\end{array}$ \\
\hline Entry into core & $\begin{array}{l}-,+ \text { or }+,- \\
-,+ \text { or }+,-\end{array}$ & $\begin{array}{l}\text { Monopolar } \\
\text { Tripolar }\end{array}$ & $\begin{array}{l}\text { Direct impact } \\
\text { Glancing impact }\end{array}$ \\
\hline Magnetosphere &,-+ & $\begin{array}{l}\text { Opposite direction of event motion } \\
\text { inferred from } B_{n} \text { signature }\end{array}$ & $V_{\text {event }}>V_{\text {sphere }}$ \\
\hline Magnetosphere &,+- & $\begin{array}{l}\text { In direction of event motion inferred } \\
\text { from } B_{n} \text { signature }\end{array}$ & $V_{\text {event }}<V_{\text {sphere }}$ \\
\hline
\end{tabular}

4. Any events during which the plasma instrument changed modes are excluded from our study.

\section{Case and statistical studies}

We begin by examining several FTEs and classifying them according to the taxonomy shown in Table 1 . We present examples of magnetospheric FTEs marked by bipolar in/out $V_{n}$ signatures and either tripolar or monopolar $V_{m}$ signatures, then examples of magnetosheath FTEs marked by bipolar out/in and in/out $V_{n}$ signatures. We then present the results of a statistical study.

\subsection{February 1994: glancing encounter with a mag- netospheric FTE}

IMP-8 observations (not shown) indicate that the IMF pointed sunward and dawnward during the interval from 13:00 to 14:00 UT on 17 February 1994. For this IMF orientation, component reconnection should occur along a line tilted from northern dawn to southern dusk that passes through the subsolar point. During this interval, Geotail was located on the equatorial pre-noon magnetopause at $\operatorname{GSM}(x, y, z)=(7.7,-6.0,-0.7) R_{E}$. Figure $6 \mathrm{a}$ and b presents Geotail magnetometer and plasma observations from 13:25 UT to 13:35 UT on 17 February 1994, respectively. The strong steady northward $\left(B_{l}>0\right)$ magnetic fields indicate that the spacecraft remained within the magnetosphere throughout this interval. Bipolar magnetic field perturbations in the $B_{n}$ component, enhanced magnetic field strengths, temperature decreases, and a density increase mark an FTE at 13:30 UT.

The sense of the bipolar magnetic field variations $(-,+)$ indicates event motion opposite the magnetic field, i.e. south-

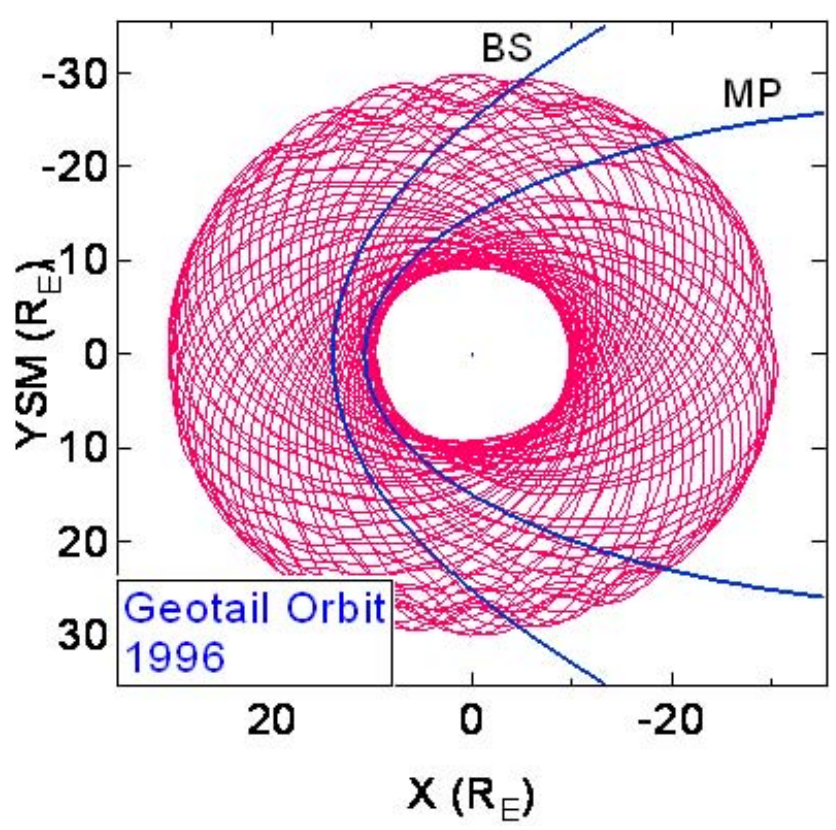

Fig. 5. Orbit of Geotail projected onto a GSM X-Y plane for 1996. The solid lines indicate positions of bow shock (BS) and magnetopause (MP).

ward away from a point of generation at the posited pre-noon reconnection line at higher northern latitudes. The $V_{l}$ and $V_{m}$ components of the velocity within the core region of the event were on the order of $50 \mathrm{~km} \mathrm{~s}^{-1}$. The magnetospheric plasma was essentially stationary. The relative flow velocity was far less than the Alfvén and sound velocities in the ambient media (1800 and $760 \mathrm{~km} \mathrm{~s}^{-1}$, respectively). Consequently, the event was moving at subsonic and subAlfvénic velocities. 
(a)

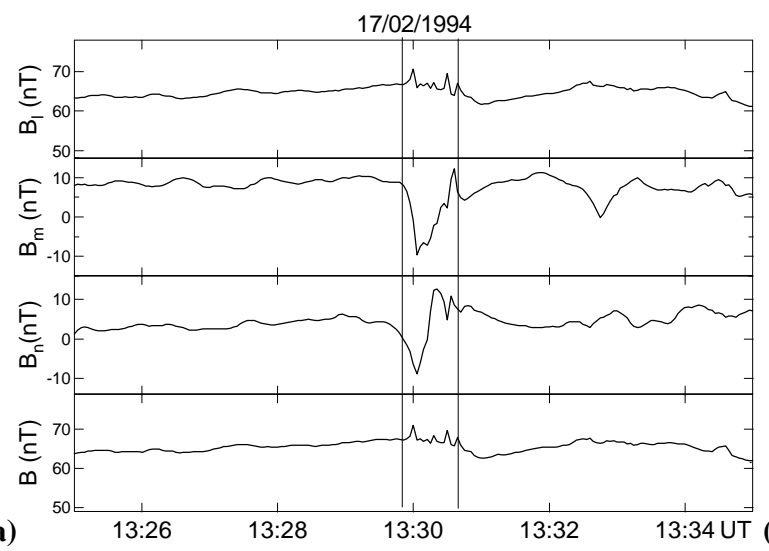

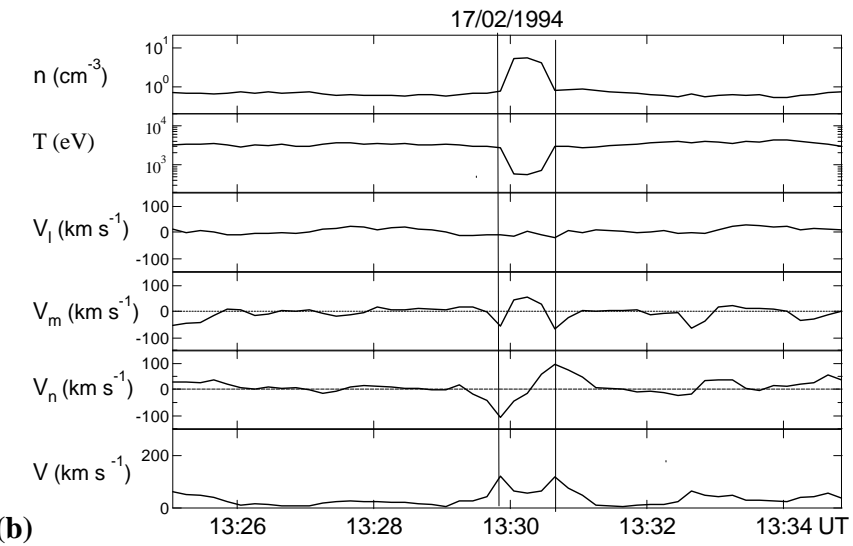

Fig. 6. Geotail (a) magnetic field and (b) ion plasma density, temperature, and velocity observations in boundary normal coordinates (1 northward, m dawnward, and n normal to the nominal magnetopause) from 13:25 UT to 13:35 UT on 17 February 1994. From top to bottom, panel (a) shows the three components of the magnetic field in boundary normal coordinates (and the strength of magnetic field. Vertical lines bound the magnetospheric FTE at 13:30 UT, which is marked by a reverse bipolar $(-,+) B_{n}$ signature, an enhanced magnetic field strength, a density increase, a temperature decrease, a tripolar $V_{m}$ signature, and an inward/outward $(-,+) V_{n}$ signature. Higher frequency compressional perturbations of the magnetic field occur at the time of this event.

We expect the magnetic field draped over events moving at subAlfvénic and subsonic velocities to rotate towards the direction perpendicular to the event axis. The magnetic field within this event rotated duskward $\left(-B_{m}\right)$. Since the IMF pointed dawnward, this rotation does not indicate entry onto a bundle of open, reconnected magnetic field lines. Instead, the duskward rotation is consistent with magnetospheric magnetic field lines draping over an event whose axis runs from northern dawn to southern dusk, i.e. parallel to the subsolar component reconnection line expected during this interval of dawnward IMF orientation.

The $V_{n}$ component exhibited a bipolar $(-,+)$ variation during the event, while the $V_{m}$ component exhibited a tripolar $(-,+,-)$ variation. According to Table 1 , these observations indicate a glancing encounter with an event moving dawnward at speeds greater than those of the background magnetospheric plasma.

Since the model predicts a density decrease for a spacecraft remaining outside an FTE moving at subsonic and subAlfvénic speeds, the large density increases and temperature decreases indicate an entry into the region of interconnected magnetosheath and magnetospheric magnetic field lines within the event. Vertical lines delimit the extent of this core region.

The continuity of the $V_{n}$ signatures across the event indicates the presence of an Alfvén wave moving along its axis in the core region. Then the positive correlation between the $(-,+) B_{n}$ and $V_{n}$ signatures indicates that this wave is moving southward, antiparallel to the $+B_{l}$ magnetic field direction. Since the reconnection site lies northward from the spacecraft, the wave is propagating from the magnetopause into the magnetosphere.

\subsection{December 1996: direct entry into a magneto- spheric FTE}

Lagged wind observations (not shown) indicate that the IMF pointed southward and duskward during the interval from 11:30 to $12: 30$ UT on 9 December 1996. For this IMF orientation, component reconnection should occur along a line tilted from southern dawn to northern dusk that passes through the subsolar point. From 12:00 to 12:10 UT, Geotail was located near the equatorial dusk magnetopause at GSM $(x, y, z)=(2.5,10.3,-2.2) R_{E}$. Figure $7 \mathrm{a}$ and $\mathrm{b}$ presents Geotail magnetic field and plasma observations for this interval. The northward $\left(B_{l}>0\right)$ and duskward $\left(B_{m}<0\right)$ magnetic fields indicate that Geotail remained within the magnetosphere throughout the period shown. A sharp decrease in the total magnetic field strength, a tripolar $B_{l}$ signature with a strong core bounded by enhanced magnetic field strengths, a bipolar $B_{n}$ signature, an increase in the density, a decrease in the temperature, and a bipolar $V_{n}$ signature indicate the passage of a crater FTE at 12:05:15 UT.

The $V_{m}$ component of the velocity within the core region of the event was on the order of $100 \mathrm{~km} \mathrm{~s}^{-1}$. The magnetospheric plasma was essentially stationary. The relative flow velocity was therefore far less than the Alfvén and sound velocities in the ambient media (1200 and $820 \mathrm{~km} \mathrm{~s}^{-1}$, respectively). Consequently, the event was moving at subsonic and subAlfvénic velocities. The sense of the bipolar magnetic field variation $(+,-)$ indicates event motion parallel to the magnetic field, i.e. northward towards (and not away from) the postulated point of generation on the northern post-noon magnetopause. Furthermore, the $B_{m}$ component diminished greatly within the event, in contrast to expectations for entry into an FTE during intervals of duskward IMF 

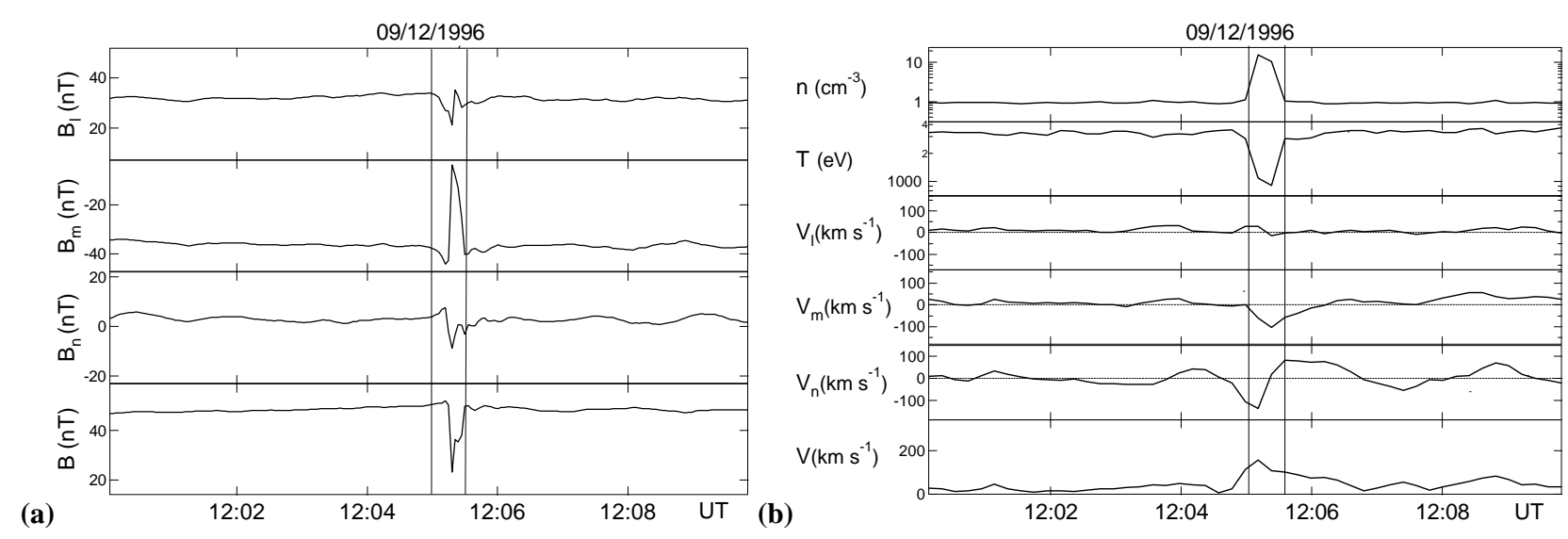

Fig. 7. Geotail (a) magnetic field and (b) ion plasma density, temperature, and velocity observations in boundary normal coordinates from 12:00 UT to 12:10 UT on 9 December 1996. The format is the same as that for Fig. 6 a and b.

orientation. Modest enhancements in the (negative) $B_{m}$ component just outside the core region of this event indicate rotation towards dusk, consistent with an event axis running from northern dawn to southern dusk. Taken together, these observations suggest that the draped magnetosheath magnetic field pointed dawnward, and not duskward as predicted on the basis of the upstream observations.

In conjunction with the weak magnetic field strengths seen at the center of this event, the strong density increase and temperature decrease indicate a direct entry into the core region. Vertical lines delimit the extent of this region. The flow vector within the core region points northward $\left(V_{l}>0\right)$ and duskward $\left(V_{m}<0\right)$ consistent with the motion inferred from the magnetic field observations. The inward/outward $(-,+)$ bipolar flow signature is consistent with that expected for an event moving faster than the background magnetospheric flow, but also with rotation inside the core region. The sense of the correlation between $B_{n}$ and $V_{n}$ indicates an Alfvén wave moving parallel to the magnetic field, also consistent with the interpretation of this event lying northward from the reconnection line. The absence of a tripolar flow signature in either the $V_{l}$ or $V_{m}$ components confirms a direct encounter.

\subsection{June 1997: a magnetosheath FTE with out- ward/inward flow}

Perigee-pass Wind observations (not shown) indicate that the IMF pointed southward and duskward during the interval from 09:00 to 10:00 UT on 12 June 1997. For this IMF orientation, component reconnection should occur along a line tilted from southern dawn to northern dusk that passes through the subsolar point. Figure $8 \mathrm{a}$ and $\mathrm{b}$ presents Geotail observations for the interval from 09:39 to 09:50 UT. A $3 \mathrm{nT}$ magnetic field and $20 \mathrm{~km} \mathrm{~s}^{-1}$ velocity component were present in the original boundary normal coordinates. To eliminate these components, the boundary normal for this interval alone was rotated $10^{\circ}$ northward and $4^{\circ}$ duskward.

The spacecraft was located in the equatorial dusk magnetosheath at GSM $(x, y, z)=(-4.5,17.9,-2.2) R_{E}$, where it observed a southward $\left(-B_{l}\right)$ and duskward $\left(-B_{m}\right)$ magnetosheath magnetic field. A flux transfer event at 09:44 UT was marked by an inward/outward bipolar magnetic field signature normal to the nominal magnetopause, enhanced magnetic field strengths, a duskward $\left(-B_{m}\right)$ and northward magnetic field rotation, depressed densities, enhanced temperatures, southward and duskward flow deflections, and a bipolar velocity signature perpendicular to the nominal magnetopause.

The simultaneous density decrease, temperature increase, and northward magnetic field rotation indicate that Geotail entered the heated core region of the event and was on magnetic field lines with orientations intermediate between those of the magnetosheath and magnetosphere. Vertical lines delimit the extent of this region. Had the spacecraft remained outside the event, adiabaticity would have dictated a simultaneous density and temperature decrease and the field would not have rotated northward. Plasma within the event flows at speeds of $320 \mathrm{~km} \mathrm{~s}^{-1}$, while the speed of the plasma outside the event is on the order of $285 \mathrm{~km} \mathrm{~s}^{-1}$. The relative velocity is therefore less than either the ambient Alfvén or sound speeds (145 and $340 \mathrm{~km} \mathrm{~s}^{-1}$, respectively) and the event is moving at subsonic and subAlfvénic velocities through the surrounding media. The southward and duskward flow direction attending the event is consistent with the southward motion of the event inferred from the $(-,+)$ bipolar magnetic field signature and Geotail's location on the equatorial magnetopause south of a reconnection line running from southern dawn to northern dusk.

The component of the velocity normal to the nominal magnetopause shows a clear positive/negative $(+,-)$ bipolar variation. According to the discussion of Fig. 1, this pattern is 
(a)

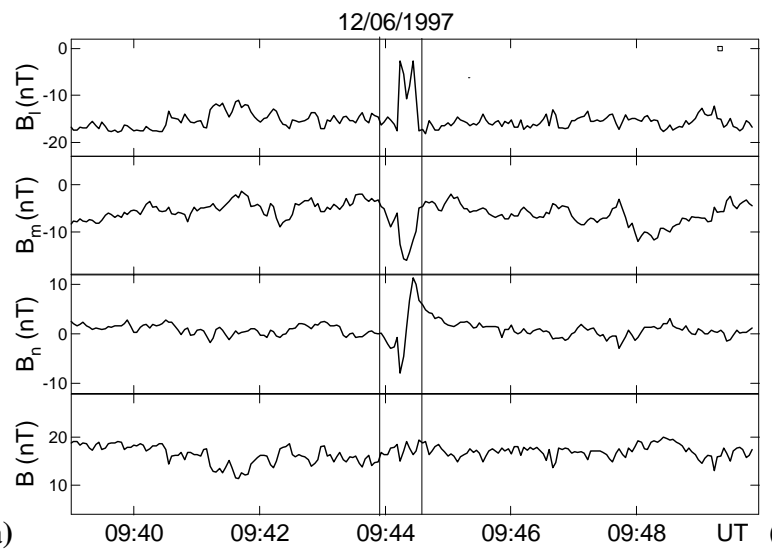

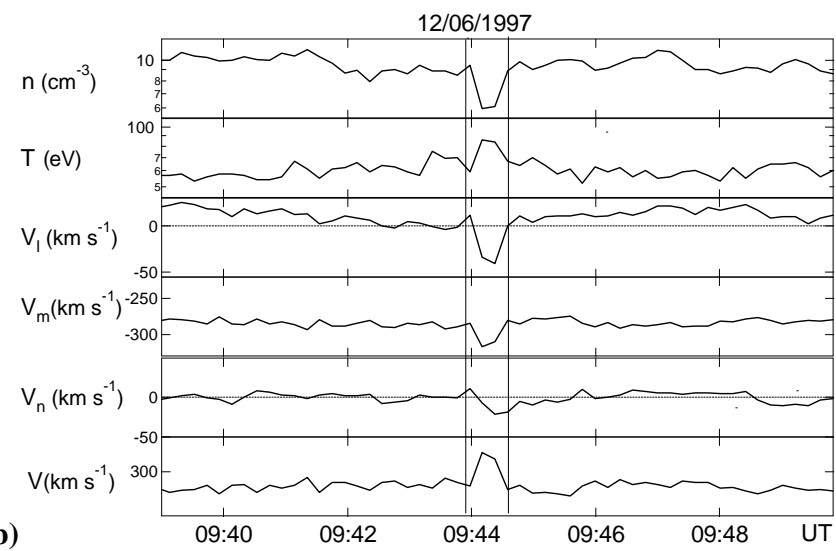

Fig. 8. Geotail (a) magnetic field and (b) ion plasma density, temperature, and velocity observations in boundary normal coordinates from 09:39 UT to 09:50 UT on 12 June 1997. The format is the same as that for Fig. 6a and b.

consistent with the perturbations expected outside an event moving faster than the surrounding flow. If a portion of this flow occurred within the core region of the event, then the anticorrelated field and flow perturbations normal to the nominal magnetopause indicate an Alfvén wave propagating parallel to the southward and duskward magnetosheath magnetic field, i.e. away from the postulated reconnection site at higher northern latitudes.

\subsection{May 1996: a magnetosheath FTE with in- ward/outward flow}

IMP-8 observations (not shown) indicate that the IMF pointed strongly southward with a fluctuating dawn/dusk component during the interval from 14:00 to 14:30 UT on 3 May 1996. For these IMF orientations, component reconnection should occur along a equatorial line passing through the subsolar point with a variable tilt. During this interval, Geotail was located in the equatorial dusk flank magnetosheath at $\operatorname{GSM}(x, y, z)=(-8.5,19.9,3.0) R_{E}$. As shown in Fig. 9a, the spacecraft observed southward $\left(B_{l}<0\right)$ magnetosheath magnetic field orientations from 14:13 to 14:25 UT. During the same interval, the component of the magnetosheath magnetic field in the dawn/dusk direction $\left(B_{m}\right)$ changed sign several times. Geotail observed an FTE at 14:21 UT marked by enhanced magnetic field strength and bipolar inward/outward $B_{n}$ magnetic field signatures. In view of the strongly southward magnetosheath magnetic field orientation, the inward/outward bipolar signature indicates an event moving southward.

Figure $9 \mathrm{~b}$ presents the corresponding plasma signatures. Densities within the event were less than those in the magnetosheath proper, while temperatures were greater.

Consequently, Geotail entered the region of interconnected magnetosheath and magnetospheric magnetic field lines within the core region of the event. Vertical lines delimit the extent of this region. Had Geotail remained outside the event, adiabaticity would have required the density and temperature to vary in phase. The strongly duskward velocities within the event core region of the event are no more than $10 \mathrm{~km} \mathrm{~s}^{-1}$ greater than those in the nearby magnetosheath. If there is any relative motion, it is at subsonic and subAlfvénic velocities.

The event exhibits a weak inward/outward $(-,+) V_{n}$ flow perturbation normal to the nominal magnetopause. According to Table 1, this signature is consistent with magnetosheath flow over an FTE moving slowly duskward. As this signature is correlated with the bipolar magnetic field signature normal to the nominal magnetopause, it can also be interpreted as evidence for an Alfvén wave propagating northward along the southward-pointing magnetosheath magnetic field. Since the weak northward $\left(+V_{l}\right)$ flows that accompanied the FTE contradict the southward motion inferred from the bipolar $(-,+) B_{n}$ signatures, the interpretation of the bipolar magnetic field and velocity signatures in terms of an Alfvén wave propagating along the event axis seems preferable. There are no clearer magnetosheath events with $(-,+)$ bipolar $V_{n}$ signatures in our database, suggesting that such events are rare.

\subsection{Statistical survey}

To determine which FTE flow velocity signatures are most prevalent, we identified 365 Geotail FTEs satisfying the magnetic field criteria described in Sect. 3 during the interval from February 1994 to June 1997. Of 213 magnetospheric events, 173 exhibited clear inward/outward $(-,+) V_{n}$ signatures, while 40 exhibited poor inward/outward $(-,+)$ or weak $V_{n}$ signatures. None exhibited outward/inward $V_{n}$ signatures. From this we draw two conclusions. First, the events invariably move with or faster than the background magnetospheric flow. Second, since the Geotail spacecraft sometimes enters the core regions of the events, the flows within this region must invariably be directed 
03/05/1996

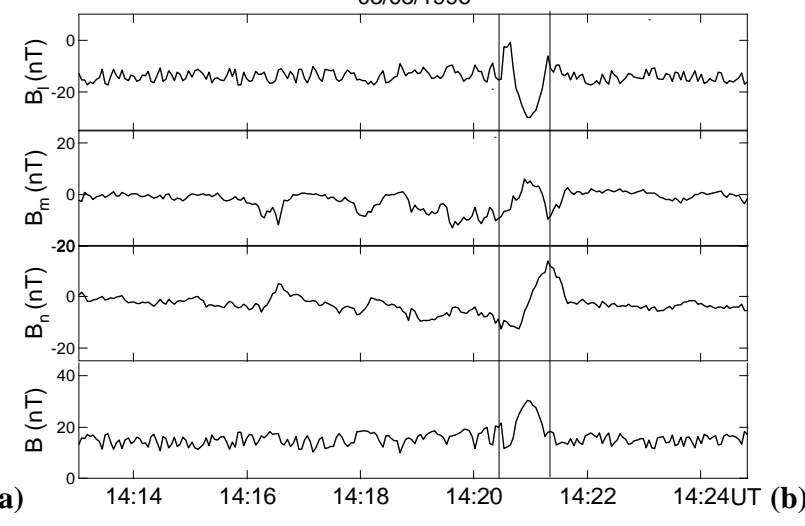

03/05/1996

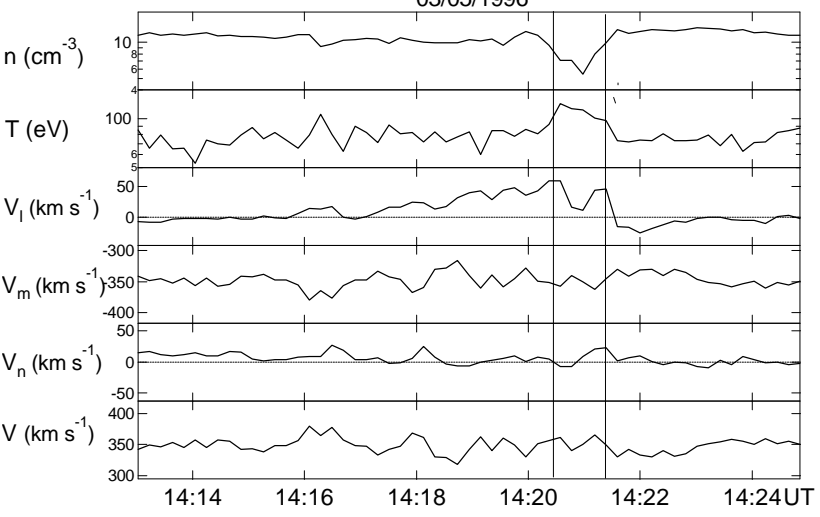

Fig. 9. Geotail (a) magnetic field and (b) ion plasma density, temperature, and velocity observations in boundary normal coordinates from 14:13 UT to 14:25 UT on 3 May 1996. The format is the same as that for Fig. $6 a$ and b.

inward/outward. Since events moving northward on the magnetopause exhibit outward/inward magnetic field signatures, the flow and field signatures within events moving northward on the magnetopause are anticorrelated and the Alfvén wave within them propagates along the magnetic field from the magnetopause into the magnetosphere. A similar line of reasoning leads to the conclusion that the Alfvén wave also propagates inward from the magnetopause to the magnetosphere in events moving southward on the magnetopause.

We found no difference in the occurrence patterns of magnetosheath and magnetospheric events. Of 152 magnetosheath FTEs, 98 displayed clear plasma flow signatures. Of these, 63 exhibited positive/negative $(+,-) V_{n}$ signatures indicating event motion faster than the background magnetosheath flow. Since (as just discussed) observations from the magnetospheric side require the sequence of internal flows seen during the passage of FTEs to be inward/outward, the spacecraft could not have penetrated the core region from the magnetosheath side during any of these 63 events. Had the spacecraft penetrated the core region, it would have observe a bipolar flow signature opposite that seen outside the event, perhaps resulting in an unclear or weak flow velocity signature normal to the nominal magnetopause. Unclear or weak signatures, indicative either of events moving with the magnetosheath flow or entries into the core region, were observed in 54 of the 152 magnetosheath events. Finally, of the 152 magnetosheath events, 35 exhibited negative/positive $(-,+) V_{n}$ flows. These flows can be interpreted as evidence either for events moving slower than the background flow or entries into events rotating in the sense determined from observations on the magnetospheric side.

\section{Summary and conclusions}

Because they displace the surrounding media, FTEs generate characteristic plasma flow velocity signatures. We discussed the flow patterns expected for events moving at subsonic and subAlfvénic velocities relative to the ambient media. Events moving opposite or in the direction of and faster than the ambient flows push the plasma ahead and entrain plasma behind, driving flows on their flanks in the direction opposite their motion. Spacecraft on paths that make glancing entries into the core region of such events may observe tripolar flow signatures: opposite event motion ahead of the events, in the direction of event motion within the events, and opposite event motion behind the events. Events moving faster than the ambient flow also generate bipolar outward/inward flows in the magnetosheath and inward/outward flows within the magnetosphere. Events moving with the ambient flow generate no flow perturbations in the ambient media. Events moving in the direction of the ambient flow, but at speeds less than that of the ambient flow, generate flow perturbations in the direction of event motion on their flanks, but opposite event motion directly ahead and behind of the events. Flows within the core region of FTEs require an interpretation in terms of Alfvénic fluctuations propagating along event axes.

We presented examples of events that excited tripolar magnetospheric flow signatures in the direction of event motion, inward/outward signatures within the magnetosphere, outward/inward signatures in the magnetosheath, and inward/outward signatures in the magnetosheath. We interpreted these events as evidence for glancing encounters with a magnetospheric event moving faster than the ambient magnetospheric flow, a direct entry into the core region of a magnetospheric event moving faster than the ambient magnetospheric flow, an encounter with a magnetosheath event moving faster than the magnetosheath flow, and an encounter with a magnetosheath event moving slower than the magnetosheath flow, respectively.

We reported the results of a preliminary statistical survey of flow signatures associated with 365 magnetospheric and magnetosheath FTEs. Magnetospheric events often exhibit inward/outward flow signatures, but never exhibit 
outward/inward flow signatures. As a result, we conclude that they invariably move at or faster than the ambient magnetospheric flow. By contrast, magnetosheath events exhibit both inward/outward and outward/inward signatures, indicating that they move both faster and slower than the ambient magnetosheath velocity. Since spacecraft frequently enter the core region of magnetospheric events, but never observe outward/inward flow signatures, the perturbations (if any) driven by Alfvénic fluctuations propagating along event axes must always be in the inward/outward sense. By comparison with the sense of magnetic field perturbations, we find that these fluctuations propagate inward into the magnetosphere from the magnetopause along open magnetic field lines.

Acknowledgements. We thank the SPDF and NSSDC at GSFC for supplying the Geotail, IMP-8 magnetic field observations. Work at GSFC was supported by NASA's Guest Investigator Program, while work by G. I. K. at the University of Maryland was supported by a grant from NASA/GSFC NNX07AH99G.

Topical Editor I. A. Daglis thanks two anonymous referees for their help in evaluating this paper.

\section{References}

Belcher, J. W. and Davis Jr., L.: Large-amplitude Alfvén waves in the interplanetary medium, 2., J. Geophys. Res., 76, 3534-3563, 1971.

Cooling, B. M. A., Owen, C. J., and Schwartz, S. J.: Role of the magnetosheath flow in determining the motion of open flux tubes, J. Geophys. Res., 106, 18 763-18 775, 2001.

Cowley, S. W. H.: The causes of convection in the Earth's magnetosphere: A review of developments during the IMS, Rev. Geophys. Space Phys., 20, 531-565, 1982.

Ding, D.-Q., Lee, L.-C., and Ma, Z.-W.: Different FTE signatures generated by the bursty single-x line reconnection and the multiple-x line reconnection at the dayside magnetopause, J. Geophys. Res., 96, 57-66, 1991.

Dungey, J. W.: Interplanetary magnetic field and auroral zones, Phys. Rev. Lett., 6, 47-48, 1961.

Farrugia, C. J., Elphic, R. C., Southwood, D. J., and Cowley, S. W. H.: Field and flow perturbations outside the reconnected field line region in flux transfer events: Theory, Planet. Space Sci., 35, 227-240, 1987

Kokubun, S., Yamamoto, T., Acuna, M., Hayashi, K., Shiokawa, K., and Kawano, H.: The Geotail magnetic field experiment, J. Geomagn. Geoelectr., 46, 7-21, 1994
Ku, H.-C. and Sibeck, D. G.: Internal structure of flux transfer events produced by the onset of merging at a single $\mathrm{X}$ line, $\mathrm{J}$. Geophys. Res., 102, 2243-2260, 1997.

Ku, H.-C. and Sibeck, D. G.: The effect of magnetosheath plasma flow on flux transfer events produced by the onset of merging, $\mathrm{J}$ Geophys. Res., 103, 6696-6702, 1998.

Lee, L.-C. and Fu, Z.-F.: A theory of magnetic flux transfer at the Earth's magnetopause, Geophys. Res. Lett., 12, 105-108, 1985.

Lockwood, M.: The ionospheric signatures of flux-transfer events and solar-wind pressure pulses, J. Geophys. Res., 95, 17113 $17135,1990$.

Mukai, T., Machida, S., Saito, Y., Hirakara, M., Terasawa, T., Kaya, N., Obara, T., Ejiri, M., and Nishida, A.: The low energy particle (LEP) experiment onboard the Geotail satellite, J. Geomagn. Geoelectr., 46, 669-692, 1994.

Rijnbeek, R. P., Cowley, S. W. H., Southwood, D. J., and Russell, C. T.: Observations of reverse polarity flux transfer events at the Earth's dayside magnetopause, Nature, 300, 23-26, 1982.

Rijnbeek, R. P., Farrugia, C. J., Southwood, D. J., Dunlop, M. W., Mier-Jedrzejewicz, W. A. C., Chaloner, C. P., Hall, D. S., and Smith, M. F.: A magnetic boundary signature within flux transfer events, Planet. Space Sci., 35, 871-878, 1987.

Roelof, E. C. and Sibeck, D. G.: Magnetopause shape as a bivariate function of interplanetary magnetic field $B_{z}$ and solar wind dynamic pressure, J. Geophys. Res., 98, 21 421-21 450, 1993.

Russell, C. T. and Elphic, R. C.: Initial ISEE magnetometer results: Magnetopause observations, Space Sci. Rev., 22, 681-715, 1978.

Saunders, M. A., Russell, C. T., and Sckopke, N.: Flux transfer events- Scale size and interior structure, Geophys. Res. Lett., 11, 131-134, 1984.

Scholer, M.: Magnetic flux transfer at the magnetopause based on single X line bursty reconnection, Geophys. Res. Lett., 15, 291294, 1988a.

Scholer, M.: Strong core magnetic fields in magnetopause flux transfer events, Geophys. Res. Lett., 15, 748-751, 1988b.

Sibeck, D. G. and Smith, M. F.: Magnetospheric plasma flows associated with boundary waves and flux transfer events, Geophys. Res. Lett., 19, 1903-1906, 1992.

Sonnerup, B. U. O.: Magnetopause reconnection rate, J. Geophys. Res., 79, 1546-1549, 1974.

Sonnerup, B. U. Ö., Hau, L.-N., and Walthour, D. W.: On steady field-aligned double-adiabatic flow, J. Geophys. Res., 97, 12 015-12 028, 1992.

Southwood, D. J., Farrugia, C. J., and Saunders, M. A.: What are flux transfer events?, Planet. Space Sci., 36, 503-508, 1988. 